\title{
Integration of data from remote monitoring systems and programmers into the hospital electronic health record system based on international standards
}

\author{
E. T. van der Velde $\cdot$ H. Foeken • T. A. Witteman • \\ L. van Erven • M. J. Schalij
}

Published online: 10 January 2012

(C) The Author(s) 2012. This article is published with open access at Springerlink.com

\begin{abstract}
Remote follow-up of implanted ICDs may offer a solution to the problem of overcrowded outpatient clinics. All major device companies have developed a remote follow-up solution. Data obtained from the remote followup systems are stored in a central database system, operated and owned by the device company and accessible for the physician or technician. However, the problem now arises that part of the patient's clinical information is stored in the local electronic health record (EHR) system in the hospital, while another part is only available in the remote monitoring database. This may potentially result in patient safety issues. Ideally all information should become available in the EHR system. IHE (Integrating the Healthcare Enterprise) is an initiative to improve the way computer systems in healthcare share information. To address the requirement of integrating remote monitoring data in the local EHR, the IHE Implantable Device Cardiac Observation (IDCO) profile has been developed. In our hospital, we have implemented the IHE IDCO profile to import data from the remote databases from two device vendors into the departmental Cardiology Information System. Data are exchanged via an HL7/XML communication protocol, as defined in the IHE IDCO profile.
\end{abstract}

Keywords Remote monitoring $\cdot$ Data exchange IHE . Standardization

\footnotetext{
E. T. van der Velde $(\bowtie) \cdot$ H. Foeken • T. A. Witteman •

L. van Erven $\cdot$ M. J. Schalij

Department of Cardiology,

Leiden University Medical Center (LUMC),

PO Box 9600, 2300 RC Leiden, the Netherlands

e-mail: ETvanderVelde@lumc.nl
}

\section{Introduction}

Implementation of the results of large randomised trials showing the effectiveness of implantable cardioverter defibrillators (ICDs) in clinical practice has led to an exponential rise in the number of implanted ICDs (Fig. 1).

The growing number of ICD recipients is resulting in a rapidly increasing workload with respect to the follow-up of these patients (Fig. 2). A regular follow-up of a pacemaker patient is scheduled every 6 months or even over a longer period. In contrast, the follow-up period of an ICD patient is usually 3-6 months. Patients with a CRT-D device (CRTICD) often require even more frequent check-ups, again leading to a greater burden on staff and time at the outpatient clinic.

Remote monitoring of implanted devices: benefits and drawbacks

Remote follow-up of implanted pacemakers or ICDs can offer a solution to the problem of overcrowded outpatient clinics, and will bring considerable convenience to the patients since they will be able to visit the outpatient clinic less frequently [1]. The clinical and health economics impact of remote monitoring, however, is still under discussion $[2,3]$. A remote monitoring system makes it possible to alternatively schedule a remote follow-up between in-clinic follow-ups. Furthermore, remote monitoring may allow early detection of ICD or lead failures without requiring any patient intervention [4] and enables early detection of arrhythmias such as atrial fibrillation or confirmation of appropriate or inappropriate shock delivery while the patient is still at home.

All major device companies have developed a remote follow-up solution. At regular intervals (depending on the 
Fig. 1 Number of ICD implantations per year (LUMC)

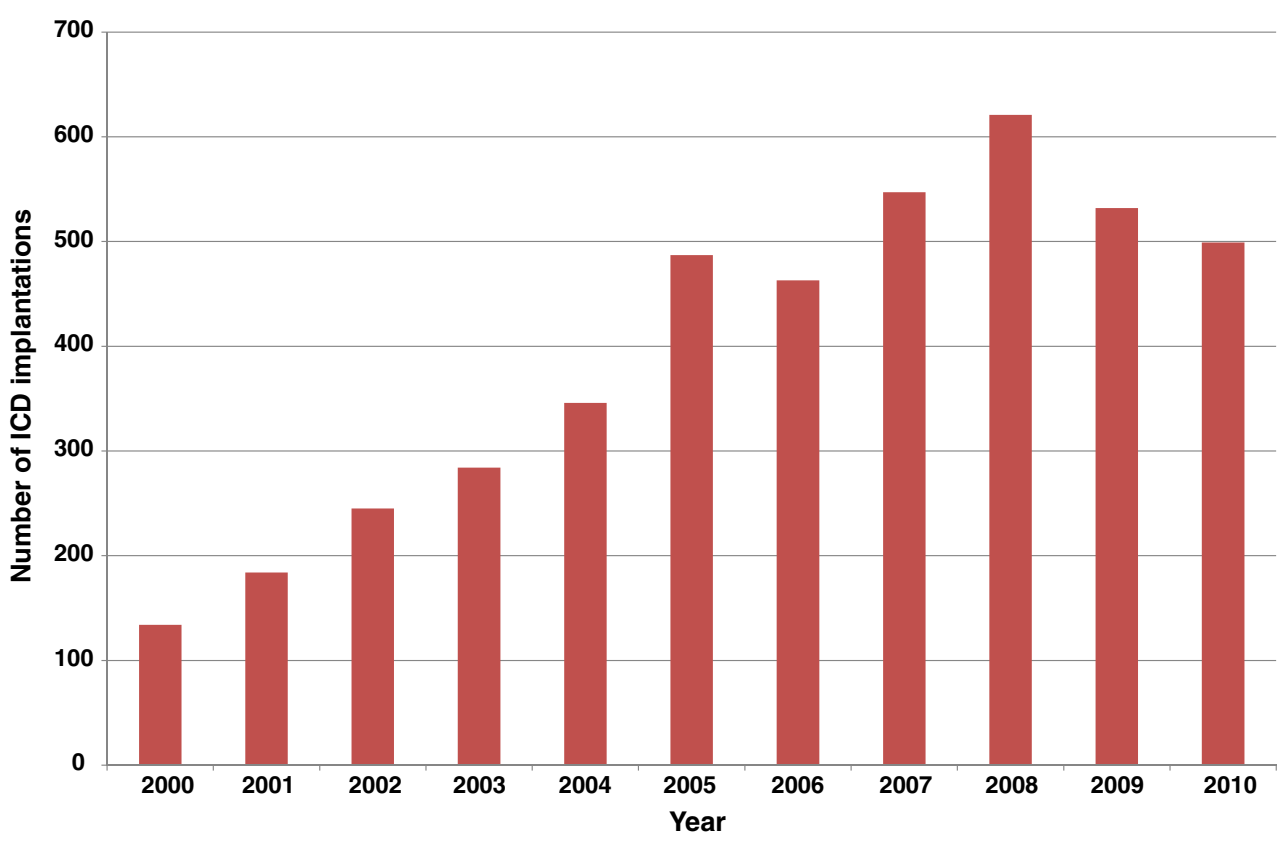

setup of the specific remote monitoring system) the implanted device will connect to a receiving device at the patient's home, and then send data on the status of the device and of the patient to the central database system, operated by the device company. The physician can log into a secure website and check the data from the remote followup for each patient. However, until now it has not been possible to integrate the data from the remote monitoring system into the local electronic health record (EHR) system. In other words data are stored in different systems and may not be accessible for all healthcare providers. This may potentially result in patient safety issues as it may be difficult to keep track of all the available information and information which may be only accessible for certain doctors or technicians. Ideally all information should become available in the EHR system.

Need for standardised data exchange

There is a need to import data from the remote monitoring database system into the local EHR. Furthermore this data import should be done in a standardised way. To obtain this goal a standard set of observations is needed, communicated in standard messages, such as: therapy settings, events, device self-monitoring. Furthermore, there should be a consistent presentation of data from all devices.
Fig. 2 Number of ICD followups per year (LUMC)

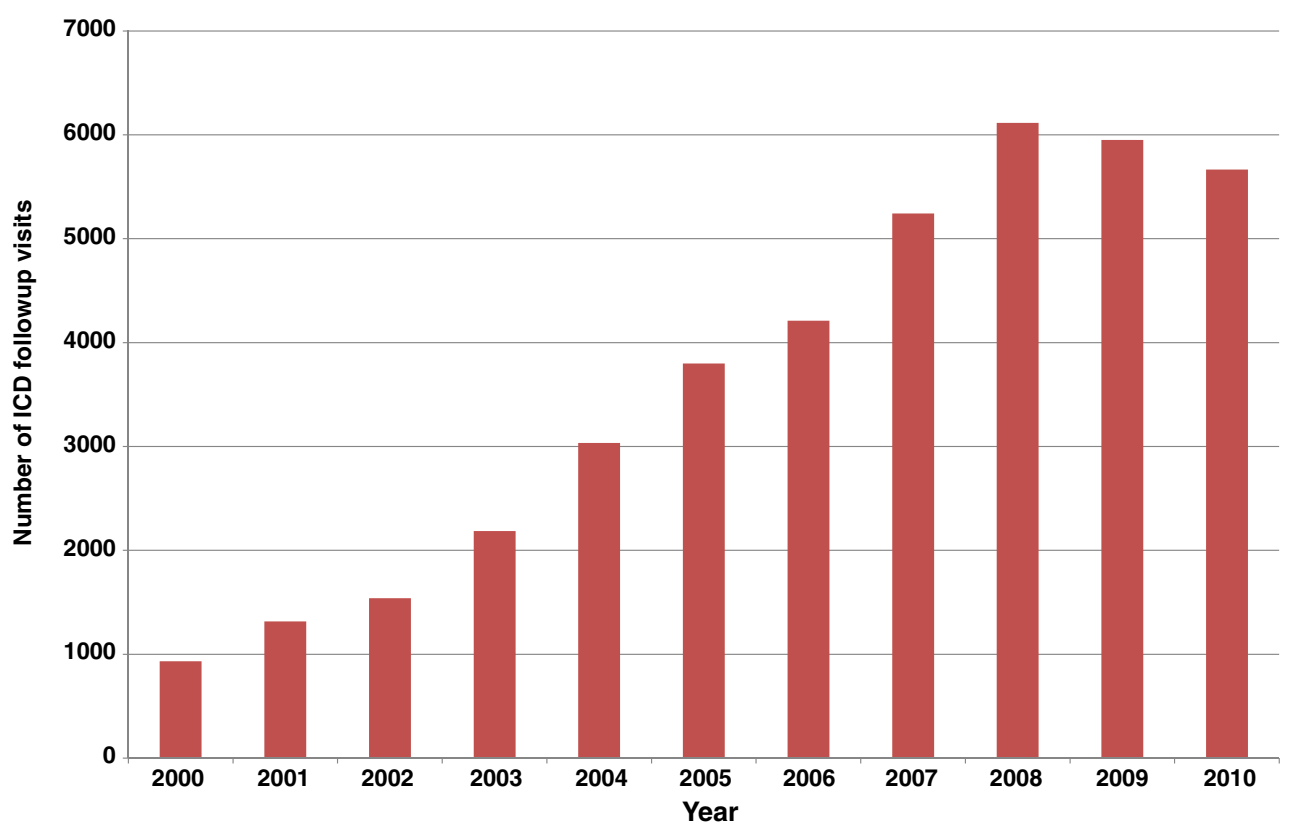




\section{Method and implementation}

Integrating the healthcare enterprise

IHE (Integrating the Healthcare Enterprise) is a shared initiative by healthcare professionals and industry to improve the way computer systems in healthcare share information. Systems that support IHE "Integration Profiles" ideally work together in a standardised way, are easier to implement, and help care providers to use information more effectively. The ultimate goal is efficient delivery of optimal patient care [5].

In various domains, IHE integration profiles specify how data can be exchanged for that specific domain and topic based on existing standards. Therefore, IHE is not a standard; it merely specifies which standards should be used in a certain domain, and how they should be used.

\section{IHE IDCO}

To address the requirement of integrating remote monitoring data in the local EHR, the IHE Implantable Device Cardiac Observation (IDCO) profile has been developed. The IHE IDCO profile defines a standard-based transfer of device interrogation information from the interrogation system into the information management system. Strong device vendor participation in the IDCO profile development is an acknowledgement of its importance. The IHE IDCO profile is part of the IHE Patient Care Devices (PCD) domain. See Fig. 3 for a schematic overview of the IHE IDCO system model.

Features of the IHE PCD IDCO profile are:

- Standard set of observations

- Communicated in standard messages

- Consistent presentation of data from all devices

- Direct link between interrogating device and local EHR

Cardiac device outpatient follow-up

The IHE-IDCO profile not only brings a solution to the problem of data in the remote monitoring database that is not available locally in the Cardiology Information System (CIS). The profile also brings a solution to the following problem. During outpatient clinic device follow-up, the measurements are performed with the use of a so-called programmer. Such a programmer system can connect wirelessly to the device implanted in the patient, and then extract the device data (e.g., settings, status, events) from the device. Furthermore, it can also be used to reprogram the settings of the device, if necessary. However, after the measurements are performed, the information needs to be
Fig. 3 IHE IDCO profile system model

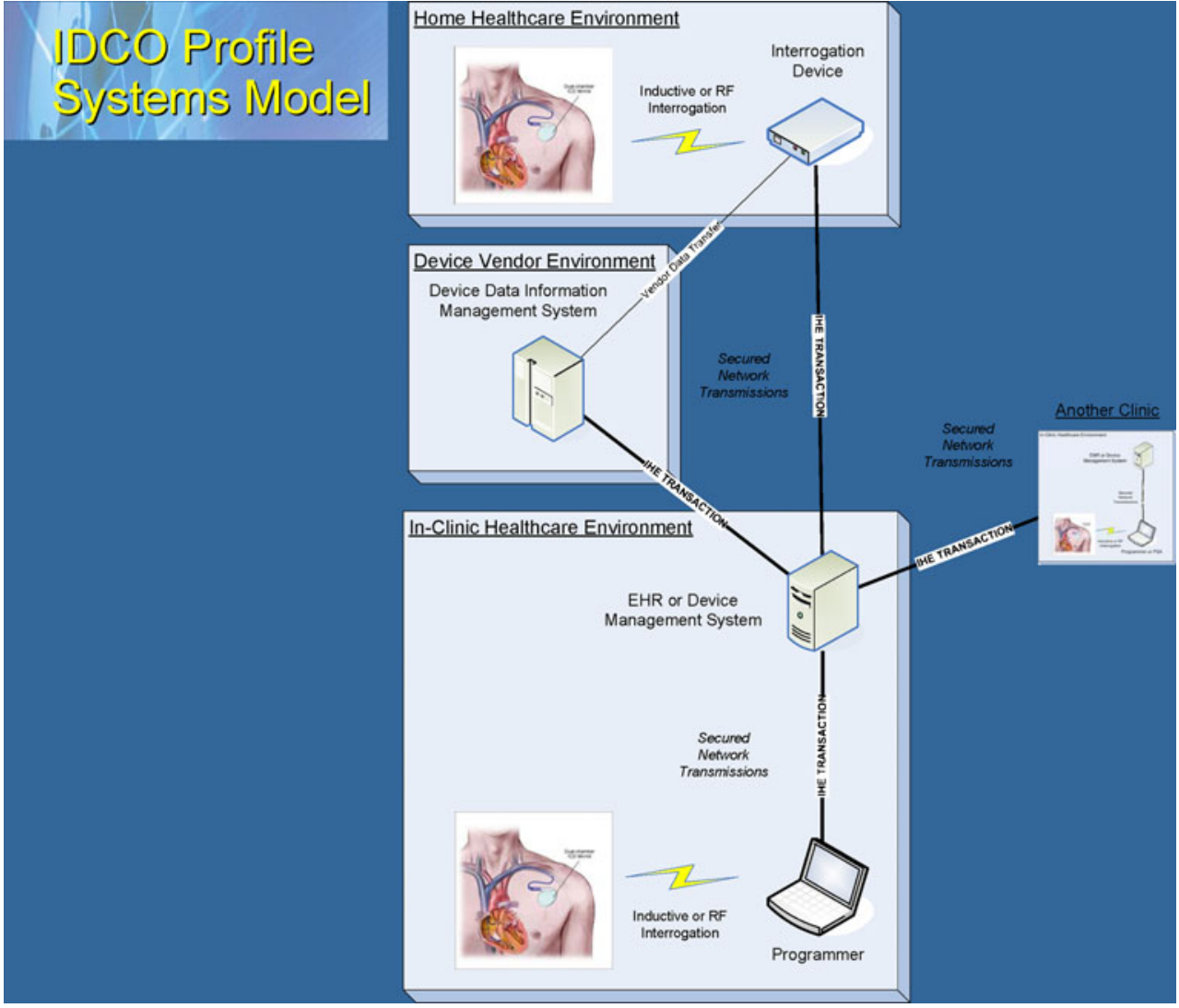


typed by hand into the CIS from a paper report printed on the programmer. The IHE IDCO profile also brings a solution to this problem, by defining standards for this specific data exchange.

\section{Nomenclature}

An important part of the IHE-PCD IDCO profile is the nomenclature, the definition of the variables that are exchanged. Companies that implement the IHE-PCD IDCO profile not only need to exchange data in a standard way, they also should make the data available using unique data definitions.

The IEEE Standards Association is defining sets of terminology for 'point-of-care' medical device communication. One of these sets is IEEE 11073-10103, which supports terminology for implantable cardiac devices. A draft version of this standard is available, but still subject to change. Representatives from all major device vendors are taking part in the IEEE 11073-10103 project group.

In Fig. 4 some of the variables as defined in the IEEE 11073-10103 standard are shown. All variable names start with 'MDC_IDC' which is short for Medical Device Communication-Implantable Device Cardiac.

Device vendor involvement and implementation

All large cardiovascular implantable device vendors are involved in the development of the IHE IDCO profile, and in the development of the IEE 11073-10103 nomenclature standard. All companies have already partially or completely implemented the IHE profile and IEEE standard and have a hardware/software solution available which can be used to communicate with an EHR or data management system.

The implementation from Biotronik, Boston Scientific and St Jude Medical is freely available, but Medtronic has only implemented the IHE IDCO profile to communicate with their proprietary solution Paceart ${ }^{\mathrm{TM}}$. Both Biotronik and St Jude Medical have also already implemented the possibility for the data exchange between a programmer and the EHR.

Implementation at Leiden University Medical Center

In our Department of Cardiology at Leiden University Medical Center, we have implemented the IHE IDCO profile to import data from the remote databases from two device vendors into the departmental Cardiology Information System (EPD-Vision ${ }^{\mathrm{TM}}$, version 8.8, Leiden University Medical Center).

Data from the remote monitoring databases from Biotronik and Boston Scientific are transferred to our Cardiology Information System, EPD-Vision ${ }^{\mathrm{TM}}$. Data are exchanged via an HL7/XML communication protocol, as defined in the IHE IDCO profile.

Data from the remote monitoring database are transferred either automatically by querying the remote database (Boston Scientific) or manually by exporting data from the remote monitoring database or programmer (Biotronik). In both cases, the data are available in the format as defined in the IEEE 11073-10103 standard.

Implementation details, EPD-Vision ${ }^{\mathrm{TM}}$

At the moment, data received from the remote monitoring system are stored in a temporary location in the EPDVision $^{\mathrm{TM}}$ database, and then become visible in EPDVision" ${ }^{\mathrm{TM}}$ in a separate ("remote monitoring") window.

The final solution is when the remote follow-up data become visible in EPD-Vision ${ }^{\mathrm{TM}}$ in the same manner as the data from the in-house follow-up. To accomplish this, we have to complete the mapping between the MDC_IDC

\begin{tabular}{|c|c|c|}
\hline Reference ID & Display Name & Definition \\
\hline MDC_IDC & Implantable Cardiac Device System & \\
\hline MDC_IDC_DEV & Implantable Cardiac Device & The group of cardiac device attributes. \\
\hline MDC_IDC_DEV_TYPE & Implantable Cardiac Device Type & The type of cardiac device. \\
\hline MDC_IDC_DEV_MODEL & Implantable Cardiac Device Model & The model identifier of a cardiac device. \\
\hline MDC_IDC_DEV_SERIAL & Implantable Cardiac Device Serial Number & The serial number of a cardiac device. \\
\hline MDC_IDC_DEV_MFG & Implantable Cardiac Device Manufacturer & The manufacturer of the cardiac device. \\
\hline MDC_IDC_DEV_IMPLANT_DT & Implantable Cardiac Device Implant Date & The implant date of the cardiac device. \\
\hline MDC_IDC_DEV_IMPLANTER & Implantable Cardiac Device Implanter & The name of the physician that implanted the cardiac device. \\
\hline MDC IDC DEV IMPLANTER CONTACT INFO & Implantable Cardiac Device Implanter Contact Infc & fc The contact information of the physician that implanted the cardiac device. \\
\hline MDC_IDC_DEV_IMPLANTING_FACILITY'- & Implantable Cardiac Device Implanting Facility & The facility (clinic / hospital) where the cardiac device was implanted. \\
\hline MDC_IDC_LEAD & Implantable Lead Attributes & The group of lead attributes. \\
\hline MDC_IDC_LEAD_MODEL & Implantable Lead Model & The model of the lead. \\
\hline MDC_IDC_LEAD_SERIAL & Implantable Lead Serial Number & The serial number of the lead. \\
\hline MDC_IDC_LEAD MFG & Implantable Lead Manufacturer & The manufacturer of the lead. \\
\hline MDC_IDC_LEAD_IMPLANT_DT & Implantable Lead Implant Date & The implant date of the lead. \\
\hline MDC_IDC_LEAD_POLARITY_TYPE & Implantable Lead Polarity Type & The number of electrodes on the lead. \\
\hline MDC_IDC_LEAD_LOCATION & Implantable Lead Location & The fixation location of the lead, usually indicating a chamber of the heart. \\
\hline MDC_IDC_LEAD_LOCATION_DETAIL_1 & Implantable Lead Location Detail 1 & The first word of a combination of words describing further details of the \\
\hline MDC_IDC_LEAD_LOCATION_DETAIL_2 & Implantable Lead Location Detail 2 & The second word of a combination of words describing further details of the \\
\hline MDC_IDC_LEAD_LOCATION_DETAIL_3 & Implantable Lead Location Detail 3 & The third word of a combination of words describing further details of the \\
\hline MDC_IDC_LEAD_CONNECTION_STATUUS & Implantable Lead Connection Status & The physical connection status of the lead, either connected or \\
\hline MDC_IDC_LEAD_SPECIAL_FUNCTION & Implantable Lead Special Function & A description of any special attribute or function of the lead. \\
\hline
\end{tabular}

Fig. 4 Some of the variables as defined in the IEEE 11073-10103 standard 
variables and the EPD-Vision ${ }^{\mathrm{TM}}$ variables, stored at the appropriate locations in the EPD-Vision ${ }^{\mathrm{TM}}$ database.

\section{Discussion and conclusion}

The growing number of ICD recipients and more complex devices (therapy platforms) are leading to a rapidly increasing workload with respect to the follow-up of these patients. All device companies have developed a system for remote monitoring of these devices. Remote monitoring will lessen the burden of follow-up on the clinic and staff; it will improve the efficiency of patient care. It is also attractive from a patient's perspective, since it may lead to greater reassurance and prevents long and time-consuming trips to the hospital.

However, as a result, part of the patient's clinical information is stored in the local electronic health record (EHR) system in the hospital, while another part is only available in the remote monitoring database. From the perspective of patient safety this is not an ideal situation.

The IHE IDCO profile has been developed to bring a solution to this problem, and to the problem that information from the interrogating devices (programmers) cannot be transferred electronically into the Electronic Health Record system.

Implementation of the IHE IDCO profile also allows for transfer of data from the interrogation device (programmer) to the local information system, which overcomes the need for manual entry of the in-house follow-up data.

In our hospital, we are currently implementing the IHE IDCO profile to store data from the remote monitoring database and programmers in our local information system (EPD-Vision $^{\mathrm{TM}}$ ). In this way, remote follow-up data can be viewed as if acquired during in-house follow-up.

An important part of the IHE IDCO profile is the nomenclature, the definition of the variables that are exchanged. The IEEE 11073-10103 standard terminology set as developed by the IEEE Standards Association is a prerequisite for easy implementation of the IHE-PCD IDCO profile. With the IEEE 11073-10103 standard set of variables, the hospital only needs to do the mapping between the dataset of the vendor and the dataset of the local Electronic Health Record system once, instead of devising a different mapping for each vendor.
The IEEE 11073-10103 standard terminology would also be very beneficial to implement in national and international cardiovascular registries, since it will allow easy data transfer from a local EHR to a national registry, and data exchange between national and international registries, such as the ESC EurObservational Research Programme [6, 7].

Future developments

It can be expected that remote monitoring systems will develop into dedicated monitoring and therapy platforms. Data retrieved from these systems should form an integral part of the electronic patient record as more and more outpatient clinic care will shift to personalised care provided at a distance, in other words in the patient's home. To accomplish such networking solutions data exchange between all the systems involved is of utmost importance. The first steps have been taken but the ultimate solution is still far ahead.

Open Access This article is distributed under the terms of the Creative Commons Attribution Noncommercial License which permits any noncommercial use, distribution, and reproduction in any medium, provided the original author(s) and source are credited.

\section{References}

1. Raatikainen MJ, Uusimaa P, van Ginneken MM, et al. Remote monitoring of implantable cardioverter defibrillator patients: a safe, time-saving, and cost-effective means for follow-up. Europace. 2008;10(10):1145-51.

2. Al-Khatib SM, Piccini JP, Knight D, et al. Remote monitoring of implantable cardioverter defibrillators versus quarterly device interrogations in clinic: results from a randomized pilot clinical trial. J Cardiovasc Electrophysiol. 2010;21(5):545-50.

3. Bikou O, Licka M, Kathoefer S, et al. Cost savings and safety of ICD remote control by telephone: a prospective, observational study. J Telemed Telecare. 2010;16(7):403-8.

4. Guedon-Moreau L, Chevalier P, Marquie C, et al. Contributions of remote monitoring to the follow-up of implantable cardioverterdefibrillator leads under advisory. Eur Heart J. 2010;31(18):224652 .

5. http://www.ihe.net

6. http://www.ncdr.nl

7. http://www.escardio.org/guidelines-surveys/eorp/Pages/welcome. aspx 